\title{
Rothmund-Thomson syndrome with esophageal stenosis: a case report
}

Keywords: poikiloderma, anal atresia, ultrasonography, Rothmund -Thomson syndrome, pyloric stenosis

\section{Introduction}

Rothmund -Thomson syndrome (RTS) is an extremely rare autosomal recessive genodermatosis. Up to now, around 300 cases were reported. Two clinical forms of RTS were described: type I, presented by Rothmund in 1863 , characterized by poikilodermatous facial rash, ectodermal dysplasia and juvenile cataracts. Type II reported by Thomson in 1923, showing poikiloderma and congenital bone defects and increased frequency of malignancy. It is caused by biallelic mutations in RECQL4, a helicase involved with chromosomal instability and DNA repair. ${ }^{1}$

Gastrointestinal abnormalities such as pyloric stenosis, anal atresia, annular pancreas and rectovaginal fistula, have also been reported. According to the literature, only two cases of RTS associated with esophageal stenosis have been described up to now., ${ }^{2,3}$ The third case of RTS associated with upper cervical esophageal stenosis is presented here.

\section{Case report}

A 23-year-old male suffers from rash over the sun exposed areas and photosensitivity started during the first months of his life. He stated that his grandfather had the same problem. No consanguinity or blood incompatibility was reported.

Skin examination demonstrated poikilodermatous rash on the face, neck (Figure 1), V zone and extensor surface of the arms and legs. Histopathology of the affected skin was consistent with poikiloderma. Eyelashes, eyebrows and scalp hair were normal. Punctate keratoderma was presented on both palms (Figure 2A). Skin biopsy taken from hyperkeratotic papules on palms showed an acanthotic epidermis with prominent granular layer, hyperkeratosis with focal parakeratosis, dilated vessels in the papillary dermis. Nails of hands (Figure 2B) and feet were dystrophic. Exophthalmos, keratoconjunctivitis sicca and blepharitis were also diagnosed. The skeletal survey was normal. Dental and neurological examinations revealed no abnormalities and his external genitalia were normal.

The patient also stated that he had severe dysphagia in childhood, and, according to medical records, barium esophagogram revealed cervical esophageal stenosis (Figure 3A). At the age of 8 , the stenosis was treated by endoscopic dilation and control esophagogram demonstrated good flow (Figure 3B). After that, he did not have a problem with dysphagia, and maintenance sessions of esophageal dilatation were not performed. Routine laboratory analyses were within normal limits. Ultrasonography of the abdomen and chest $\mathrm{X}-$ ray examinations did not show any abnormalities. The patient refused karyotype analysis.

\section{Discussion}

RTS is a rare disease and because of its broad clinical spectrum, which includes some common features, as well as some individual

\author{
Volume 2 Issue 2 - 2018 \\ Jelica Vukicevic \\ School of Medicine, University of Belgrade, Serbia
}

Correspondence: Jelica Vukicevic, School of Medicine, University of Belgrade, Belgrade, Serbia Clinic of Dermatovenereology, Clinical center of Serbia, Pasterova 2, Belgrade, Serbia, Tel +38I I I 2642648 ,

Email jvukicevicsretenovic@gmail.com

Received: February 16, 2018 | Published: April 04, 2018

reports on clinical manifestations and abnormalities, patients can be easily misdiagnosed (4).The presented patient, according to the skin, nails and ocular manifestations, and despite the fact that he has not had the cataract, could be classified as type I of RTS. Gene sequencing with mutations in both alleles of the RECQL4 helicase gene is typical for type II of the disease. The same mutations have been identified in Baller Gerold syndrome and RAPADILINO syndrome as well. In RAPADILINO syndrome, poikiloderma as the main cutaneous sign of RTS is absent. On the other hand, the most common and defining features of Baller-Gerold syndrome are cranyosinostosis and radial ray deficiency. Association of RTS and esophageal stenosis is very rare, and to the best of our knowledge, this is the third published case in the English literature.

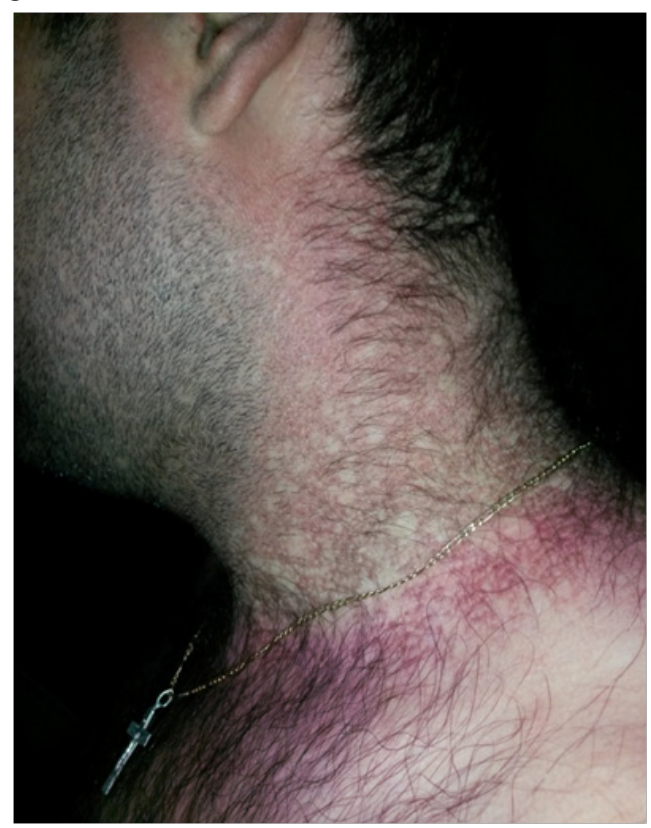

Figure I Poikiloderma on the neck. 

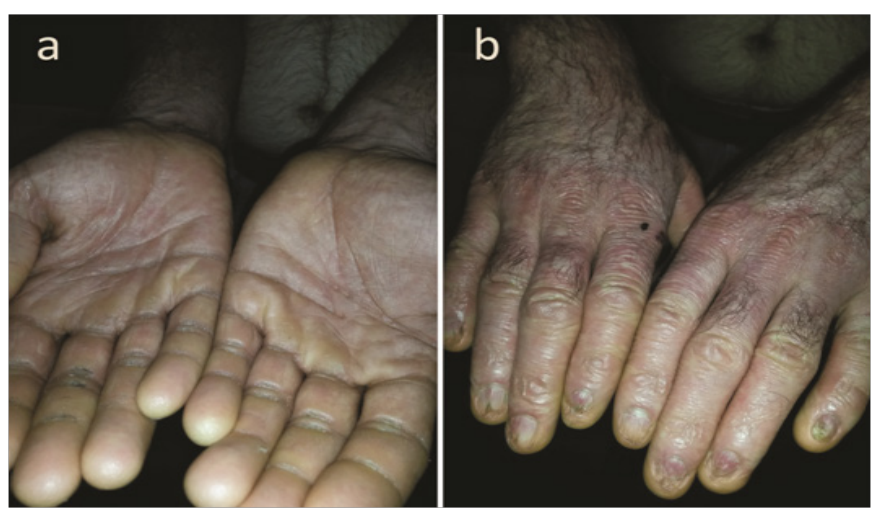

Figure 2 Palmar keratoderma and nails dystrophy.

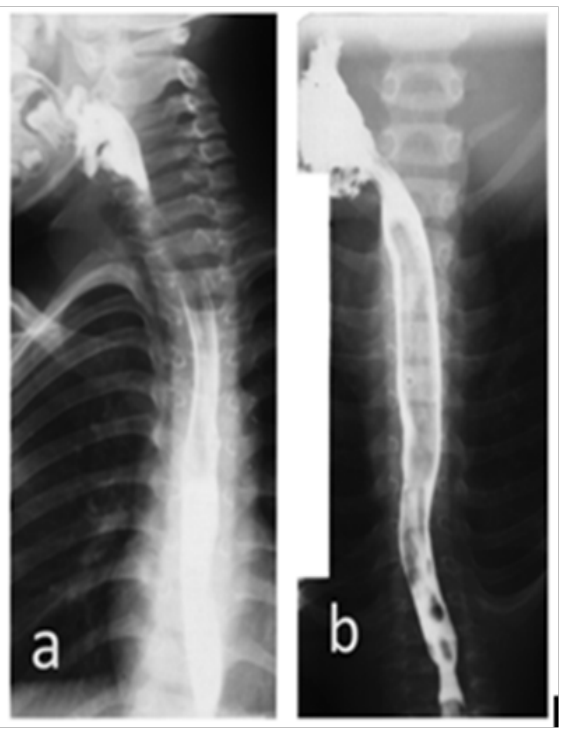

Figure 3 Barium esophagogram before (a) and after (b) dilation.

Polese L et al. ${ }^{3}$ presented RTS with cervical esophageal stenosis and iron-deficiency, which were improved by endoscopic dilation, although yearly maintenance sessions are required. Because of the inflammatory nature of stenosis, authors were able to exclude its genetic origin. ${ }^{3}$ The first case of RTS and esophageal stenosis was described by Guler O, and coworkers in $1998 .{ }^{2}$

Endoscopic dilation seems to be a very effective treatment for esophageal stenosis. ${ }^{3}$ The presented patient denied any more problems with dysphagia and maintenance endoscopic dilations were not performed. Because the ectodermal compartment (skin, hair, nails, and teeth) is the first to be affected, the main clinical diagnostic criterion for RTS is cutaneous rash. Skin lesions develop during the first three years of life, involving cheeks, and then spread to the extremities. In the acute phase lesions are in the form of erythematous and edematous patches, at times with blistering, which in chronic phase have become poikilodermatous.

Palmoplantar keratoderma has also been shown. ${ }^{4}$ Popadic et al. ${ }^{5}$ presented the first case of RTS and plantar keratoderma. ${ }^{5}$ Nail dystrophy could be an associated disease, such as in the presented case. Hair is commonly affected. Scalp hair is brittle or missing, while eyelashes and/or eyebrows are diminished. Dental anomalies incidence has been estimated at between $27 \%$ and $59 \%$ of cases.
Other heterogeneous features include short stature, ophthalmic abnormalities (bilateral cataract, keratoconus, exophthalmos, temporetinal degeneration, glaucoma, prominent Schwalbe's line, absence of mesodermal layer of iris, tilted optic discs, pigment deposits over cornea and conjunctiva), skeletal abnormalities (frontal bossing, saddle nose, short stubby fingers, congenital radial ray defects), predisposition to malignancies (osteosarcoma, Hodgkin's disease, myelodysplasias) or skin cancers (squamous cell carcinoma, basal cell carcinoma, malignant fibrous histiocytoma). ${ }^{6}$

Rare cases of RTS with bronchiectasis and atypical meningioma have been reported. ${ }^{6,7}$ In spite of the fact that there is a wide spectrum of abnormalities that could be involved in RTS, the diagnosis is commonly based on clinical and radiological examinations. Differential diagnosis must be made taking into consideration other syndromes with some identical signs such as childhood poikiloderma (eg. Werner, Cockayne, Bloom, Gottron's syndrome, ataxia telangiectasia). Kindler syndrome is, also, congenital poikiloderma syndrome that could be associated with esophageal stenosis. Otherwise, it is characterized by trauma-induced acral bullae which appear at birth or during the first few days of life (like hereditary dystrophic epidermolysis bullosa). Furthermore, developing poikiloderma and skin atrophy affect sunexposed and non-sun-exposed skin. ${ }^{1}$

A rare case of RTS with poikiloderma, exophthalmos, palmar keratoderma, nail dystrophy, esophageal stenosis is presented, and even though dermatologic screening and radiographic assessment were performed with no evidence of neoplastic diseases, continual screening for malignancy is suggested.

\section{Acknowledgements}

None.

\section{Conflicts of interest}

The authors declared that there are no conflicts of interest.

\section{References}

1. Larizza L, Roversi G, Volpi L. Rothmund-Thomson syndrome. Orphanet J Rare Dis. 2010;5:2.

2. Güler $\mathrm{O}$, Aydin $\mathrm{M}$, Uğraş $\mathrm{S}$, et al. Rothmund Thomson syndrome associated with esophageal stenosis: report of a case. Surg Today. 1998;28(8):839-842.

3. Polese S, Merigliano B, Mungo G, et al. Report on a case of RothmundThomson syndrome associated with esophageal stenosis. Dis Esophagus. 2011;24(8):E41-E44.

4. Guerrero- Gonzälez GA, Hernändez-Juärez AA, Espinoza-Gonzälez NA, et al. Rothmund-Thomson syndrome: a 13-year follow up. Case Rep Dermatol. 2014;6(2):176-179.

5. Popadic S, Nikolić M, Gajić-Veljić M, et al. Rothmund-Thomson syndrome: The first case with plantar keratoderma and the second with coeliac disease. Acta Dermatovenerol Alp Pannonica Adriat. 2006;15(2):90-93.

6. Mahajan VK, Sharma V, Chauhan PS, et al. Rothmun-Thomson syndrome with bronchiectasis: An uncommon phenotype? Indian $J$ Dermatol Venereol Leprol. 2015;81(2):190-192.

7. Pencovich N, Margalit N, Constantini S. Atypical meningioma as a solitary malignancy in a patient with Rothmund-Thomson syndrome. Surg Neurol Int. 2012;3:148. 